\title{
Die lithostratigraphische Gliederung der Ablagerungen seit der letzten Zwischeneiszeit
}

\section{Einleitung}

Es ist nicht möglich, im Rahmen dieses Heftes eine umfassende Geologie der letzten Eiszeit im nördlichen Alpenvorland der Schweiz darzulegen. Eine solche Darstellung liegt ja bereits vor und ist anderweitig veröffentlicht worden (HANTKE, 1978, 1980). Wir möchten uns im folgenden darauf beschränken, die bis heute erarbeiteten stratigraphischen Gliederungsmöglichkeiten der letzten Eiszeit, also weniger Einzelheiten als vielmehr Grundlagen darzustellen, und zwar möglichst ohne «korrelative Entwicklungshilfe» zu beanspruchen. Es geht uns darum zu zeigen, welche Möglichkeiten einer lithogenetisch-stratigraphischen Gliederung, dargestellt an ausgewählten Schlüsselprofilen, gegeben sind. Dabei werden wir auf eine regionale Letzt-Eiszeitstratigraphie Bezug nehmen, ohne durch weitreichende Korrelationen klimastratigraphische Schlußfolgerungen zu präjudizieren.

Wir haben es gewissermaßen glücklichen Umständen zu verdanken, daß wir zu dieser Arbeitsweise veranlaßt werden: als Ergänzung zu den Arbeiten von Professor welten im nördlichen Alpenvorland der Schweiz werden lithostratigraphische Untersuchungen sinnvoll und notwendig. Auch wenn vielleicht zum jetzigen Zeitpunkt Quartärpalynologie und -geologie noch nicht ganz die gleiche Sprache sprechen und wahrscheinlich auch noch nicht in gleichen stratigraphischen Maßstäben denken, so liegt dennoch gerade in einer solchen Zusammenarbeit der konstruktive Ansporn, um die Arbeit auf dem Weg zu einer Verbesserung der Eiszeitstratigraphie fortzusetzen.

Im folgenden gestatten wir uns, nochmals von den Verhältnissen im Berner Mittelland auszugehen und von dort her auf die lithogenetischen Grundzüge der letzten Eiszeit hinzuweisen. - Wir werden diesmal nicht auf die Ausdehnung der letzteiszeitlichen Gletscher eingehen, denn diese Diskussion ist Gegenstand einer besonderen, ergänzenden Darstellung (schlüchter, im Druck). Ebenso geht es in dieser Überblicksdarstellung nicht um eine detaillierte Charakterisierung der ausgeschiedenen stratigraphischen Einheiten; es sei gestattet, für solche Fragen auf bestehende Veröffentlichungen $\mathrm{zu}$ verweisen (sCHLÜCHTER, 1973, 1975, 1976).

\section{Der letztinterglaziale Bezugshorizont}

Der Nachweis echt letztinterglazialer Ablagerungen ist meistens komplexer Natur und nicht immer unzweideutig. Entweder sprechen bei palynostratigraphischer Beweisführung lithostratigraphische Argumente dagegen, oder umgekehrt; abgesehen davon, daß der Nachweis der letzten $Z$ wischeneiszeit nach rein lithologischen Kriterien nicht möglich ist. Nur eine kombinierte, sich ergänzende litho- und biostratigraphische Argumentation kann zu einer schlüssigen klimastratigraphischen Synthese führen.

Im Aarequerschnitt des Mittellandes haben wir doch einige Profile, wo letztinterglaziale Bildungen nachgewiesen sind und wo somit ein praktischer Referenzhorizont für die stratigraphische Gliederung der jüngeren Ablagerungen gegeben ist.

Das hier diskutierte stratigraphische Bezugsniveau basiert auf der palynostratigraphischen Definition des letzten Interglazials im nördlichen Alpenvorland der Schweiz durch wELTEN und geht forschungsgeschichtlich einerseits auf die Bearbeitung des Thungschneitprofils, andererseits auf jenes der Wässerifluh (Glütschtal) zurück (MÜLLER-BECK, 1959; WELTEN, 1962; SCHLÜCHTER, 1972, 1973). Im engeren Untersuchungsgebiet kommt das Profil Thalgut hinzu (sCHLÜCHTER, 1976). In neuerer Zeit sind diese lokalen Profile mit den Ergebnissen von Meikirch und aus dem Zürcher Oberland (wELTEN, 1981) in einen überregionalen Zusammenhang gestellt worden. Auf die Diskussion, ob das von wELTEN definierte EemInterglazial chronostratigraphisch dem $\mathrm{R}$ iss/WürmInterglazial der Typusregion entspricht, soll in diesem Zusammenhang nicht eingegangen werden. Diese Frage ist anderweitig erörtert worden (SCHLÜCHTER, im Druck). Der palyno-/klimastratigraphische Nachweis Eem-interglazialer Ablagerungen (sensu wELTEN) und die hier vorläufig noch "sinnvolle Korrelation» von Eem, Riss/Würm-Interglazial und der Isotopenstufe $5 e$ ergibt somit die zeitliche und landschaftsgenetische Basis für die Diskussion der geologischen

Dr. Christian Schlüchter

Institut für Grundbau und Bodenmechanik ETH-Hönggerberg, 8093 Zürich 


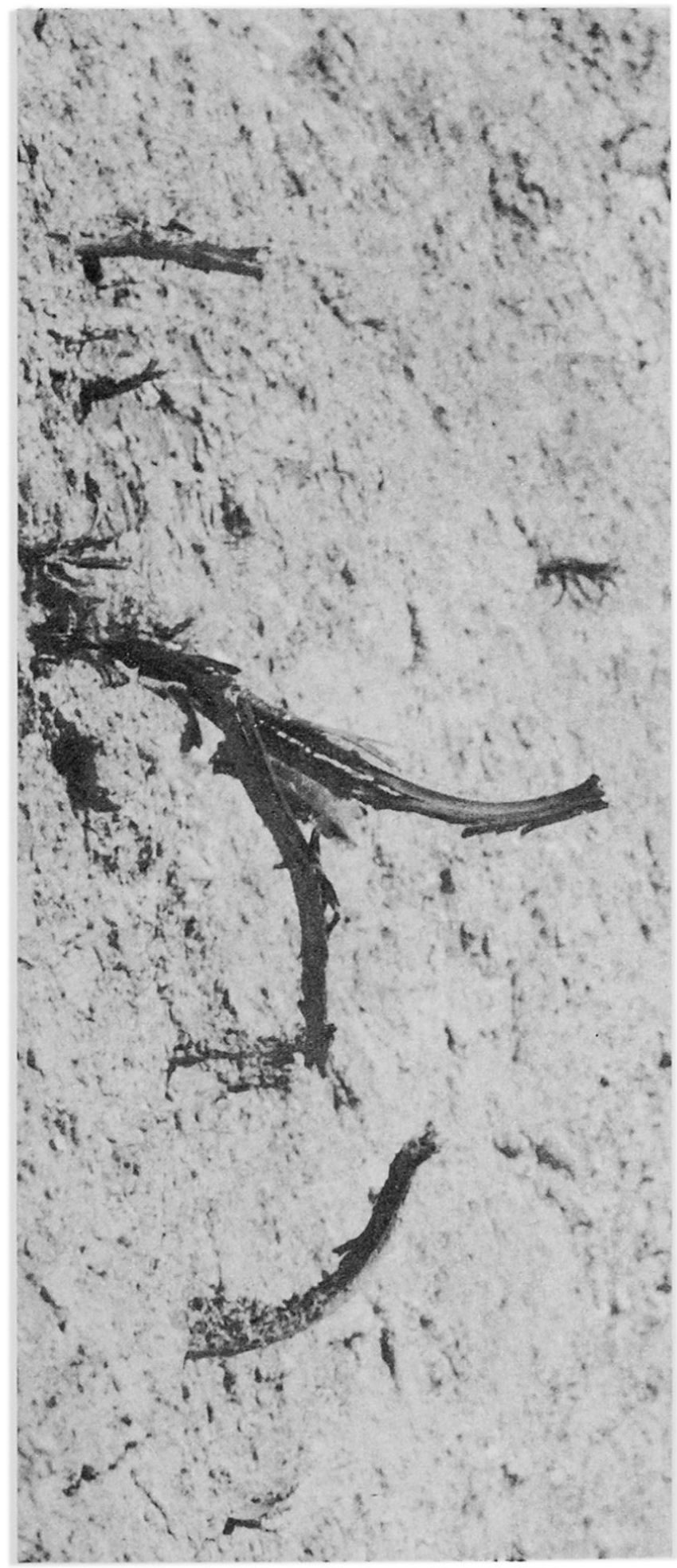

Abb.1 Detailaufnahme der Kiesgrubenwand im Thalgut mit aus den Seetonen herausragenden fossilen und gepreßten "Laden" (Profilausschnitt im Vordergrund - ca. $1 \mathrm{~m}$ ).

Ereignisse der letzten Eiszeit. Nach dem PENCK \& BRÜCKNERSchen System (1909) ist diese letzte (= Würm-) Eiszeit einphasig verlaufen, eine Auffassung, die seither schon mehrmals «aufgegliedert» worden ist. Um diese Aufgliederung im stratigraphischen und regionalen Sinne soll es im folgenden gehen.
Durch Ablagerungen belegt ist die letzte Zwischeneiszeit (Eem nach weltens Palynostratigraphie) im Aarequerschnitt mit zwei Profilen im Glütschtal (Wässerifluh und Bärebode), dem Thungschneitprofil bei Heimberg, dem Thalgut und möglicherweise bei Kienersrüti, wobei dort die lithostratigraphische Situation unklar ist (DIEGEL, 1975: 524). Es handelt sich bei diesen Profilabschnitten durchwegs um relativ geringmächtige Seeablagerungen (oder im Falle von Kienersrüti um den obersten Teil einer feinkörnigen Abfolge): um feingeschichtete Beckentone, die infolge der nachträglichen Vorbelastung durch den Gletscher stark kompaktiert sind. Aus der Verbreitung

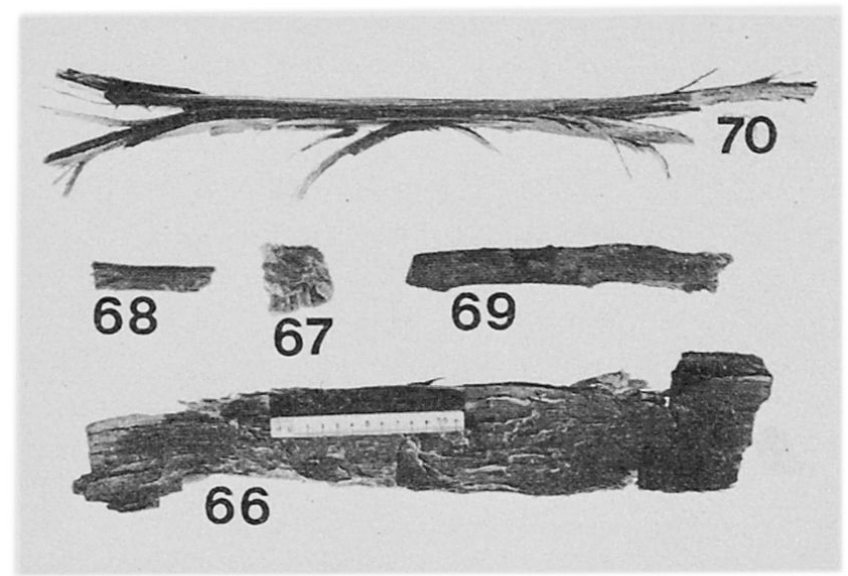

Abb. 2 Gepreßte Holzstücke aus den Thalgut-Seetonen: $66=$ Nadelholz, $67=$ Abies, $68=$ Picea, $69=$ Laubholz, $70=$ Picea (nach der freundlichen Bestimmung von PD Dr. F. Schweingruber).

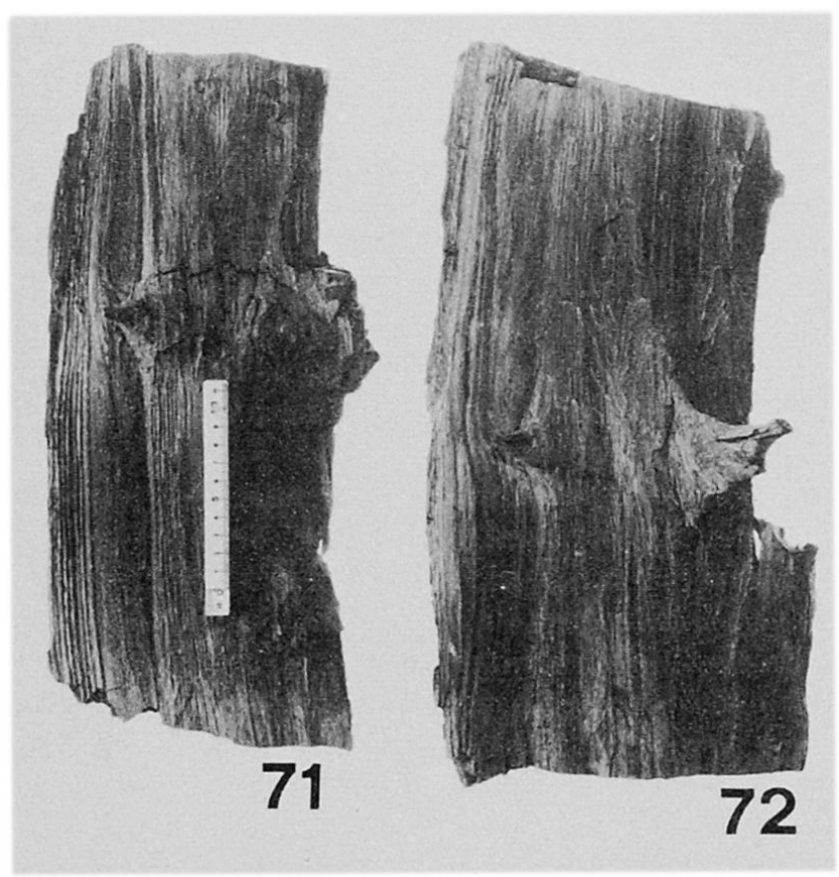

Abb. 3 Gepreßte "Laden" aus den Thalgut-Seetonen: $71=$ Abies, $72=$ Abies (nach der freundlichen Bestimmung von PD Dr. F. Schweingruber). 
und der Fazies dieser Vorkommen geht größtenteils hervor, daß die Seen, in welchen diese Seetone zur Ablagerung gekommen sind, wahrscheinlich nicht ausgedehnte Gewässer gewesen sind, sondern am ehesten mit dem heutigen Amsoldinger- oder Gerzensee verglichen werden könnten. Vor allem in den Profilen Thalgut und Thungschneit zeichnen sich diese Seetone durch großen Fossilreichtum aus (sCHLÜCHTER, 1973, 1976 und Abb. 1 bis 4). Interessant ist das reichliche Auftreten von gepreßten Holzstücken («Laden»), vor allem im Thalgut (Abb. 1 bis 3): bis jetzt konnten weit über 100 Proben geborgen werden, die teilweise mit der Radiokarbonmethode datiert worden sind. Es ist in diesem Zusammenhang angebracht, die Problematik um die Thalgut-Datierungen kurz zu diskutieren.

Die ursprünglich entnommenen Holzproben (Streuproben) haben die bereits früher mitgeteilten Alter ergeben:

$\mathrm{CS}-\mathrm{Th}-12=\mathrm{B}-2366=19530 \pm 200$ y BP

$\mathrm{CS}-\mathrm{Th}-4=\mathrm{B}-2342=28300 \pm 600$ y BP

CS - Th $-5=\mathrm{B}-2340=\geqq 39000$ y BP.

Während der Vorbereitungsarbeiten für die IGCPExkursionstagung sind 1975/76 weitere, eingemessene Proben entnommen worden, welche die folgenden, stratigraphisch logischen Alterswerte ergeben haben (die stratigraphisch höchste Probe als jüngste, die stratigraphisch tiefste als älteste):

$\mathrm{CS}-\mathrm{Th}-41=\mathrm{B}-2751=33130+1000 /-890$ y BP

$\mathrm{CS}-\mathrm{Th}-42=\mathrm{B}-2752=34300+915 /-815$ y BP

$\mathrm{CS}-\mathrm{Th}-43=\mathrm{B}-2753=36850+2050 /-1640$ y BP

CS $-\mathrm{Th}-44=\mathrm{B}-2754=43790+4360 /-2810$ y BP.

Da die Probe CS - Th - 12 mit dem Alter von $19530 \pm 200$ y BP natürlich von chronostratigraphi- schem Interesse gewesen ist, wurde sie im Zusammenhang mit Deuteriumsanalysen am Institut für Radiohydrometrie, München, ${ }^{14} \mathrm{C}$-nachdatiert. Dabei sind die folgenden Alter gemessen worden:

$$
\begin{aligned}
\mathrm{CS}-\mathrm{Th}-12=\text { München (A) } & =50000 \pm 4500 \text { y BP } \\
\text { (B) } & =>48000 \text { y BP. }
\end{aligned}
$$

Weitere Nachdatierungen der gleichen Probe in Bern haben zusätzlich die folgenden klärenden Messungen erbracht:

$$
\begin{aligned}
& \text { CS }- \text { Th }-12=\text { B }-2366=\text { Nachdatierung } \\
& \text { (A) }=>45000 \text { y BP } \\
& \text { (B) }=44000 \pm 1000 \text { y BP. }
\end{aligned}
$$

Zusätzlich sind in Bern noch die folgenden Nachbzw. Neudatierungen vorgenommen worden:

$$
\begin{aligned}
& \mathrm{CS}-\mathrm{Th}-5=\mathrm{B}-2340=\text { Nachdatierung }= \\
& >55000 \text { y } \mathrm{BP} \\
& \mathrm{CS}-\mathrm{Th}-33=\mathrm{B}-2366 \mathrm{a}=\text { Neudatierung }= \\
& >56000 \text { y BP. }
\end{aligned}
$$

Gleichzeitig mit den Neu- und Nachdatierungen der Thalgutproben ist dann durch die Erweiterung der Kiesgrube die Gliederbarkeit der Ablagerungen im Hangenden der Seetone ersichtlich geworden, und die Quelle der Verunreinigung (= der Verjüngung) der Probenalter kann nun durch die Bodenbildung im Hangenden der unteren letzteiszeitlichen Akkumulation sinnvoll erklärt werden. Die Quintessenz aus der Thalgut-Altersdiskussion ist die Erkenntnis, daß die datierten Hölzer, und somit auch die Seetone, älter als 56000 y BP sind und daß die jungen Alter möglicherweise die Bodenbildung im Hangenden datiert haben. - Die weiteren, 1976 ausführlich dargestellten Kriterien, die aus einer Interpretation des Fossilinhaltes abgeleitet werden (inkl. Paläomagnetik) und die für

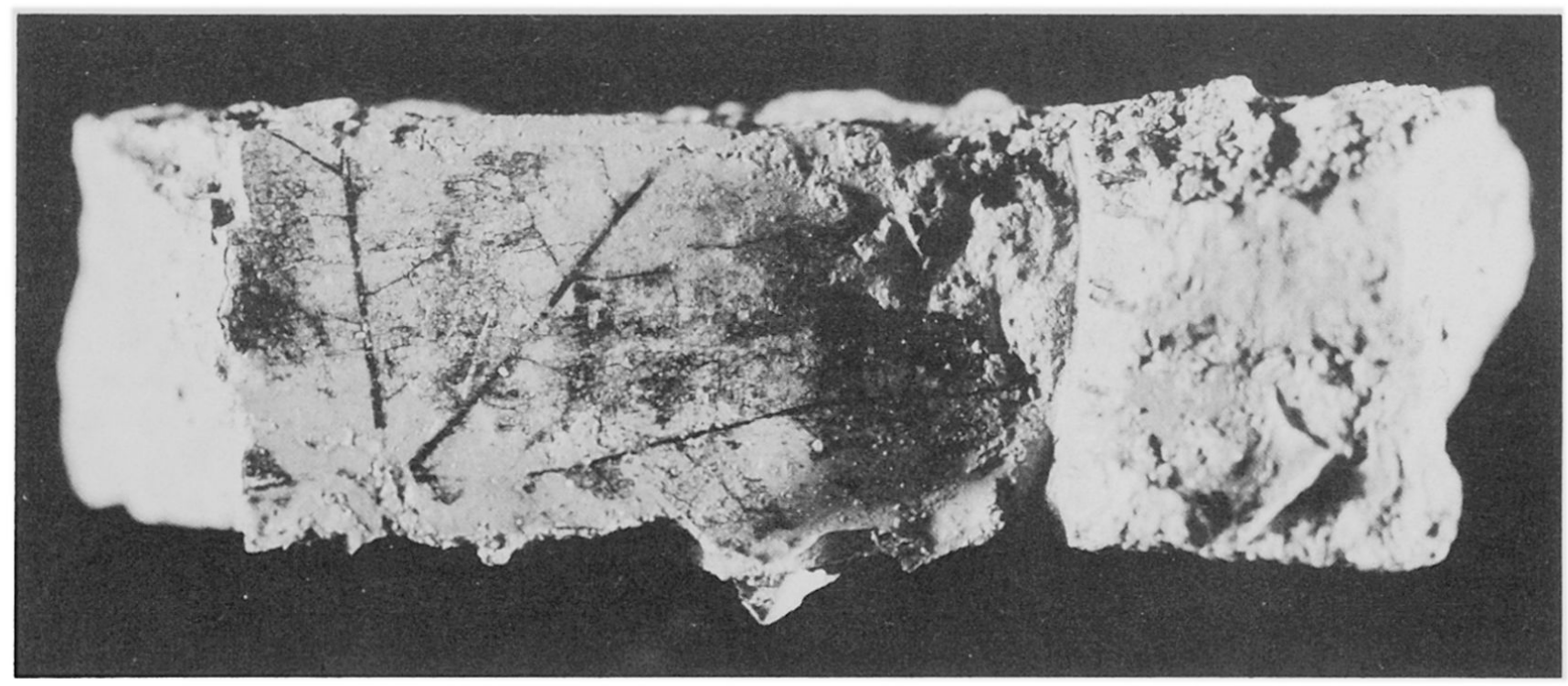

Abb. 4 Blattrest von Acer sp. aus den Thungschneit-Seetonen. 


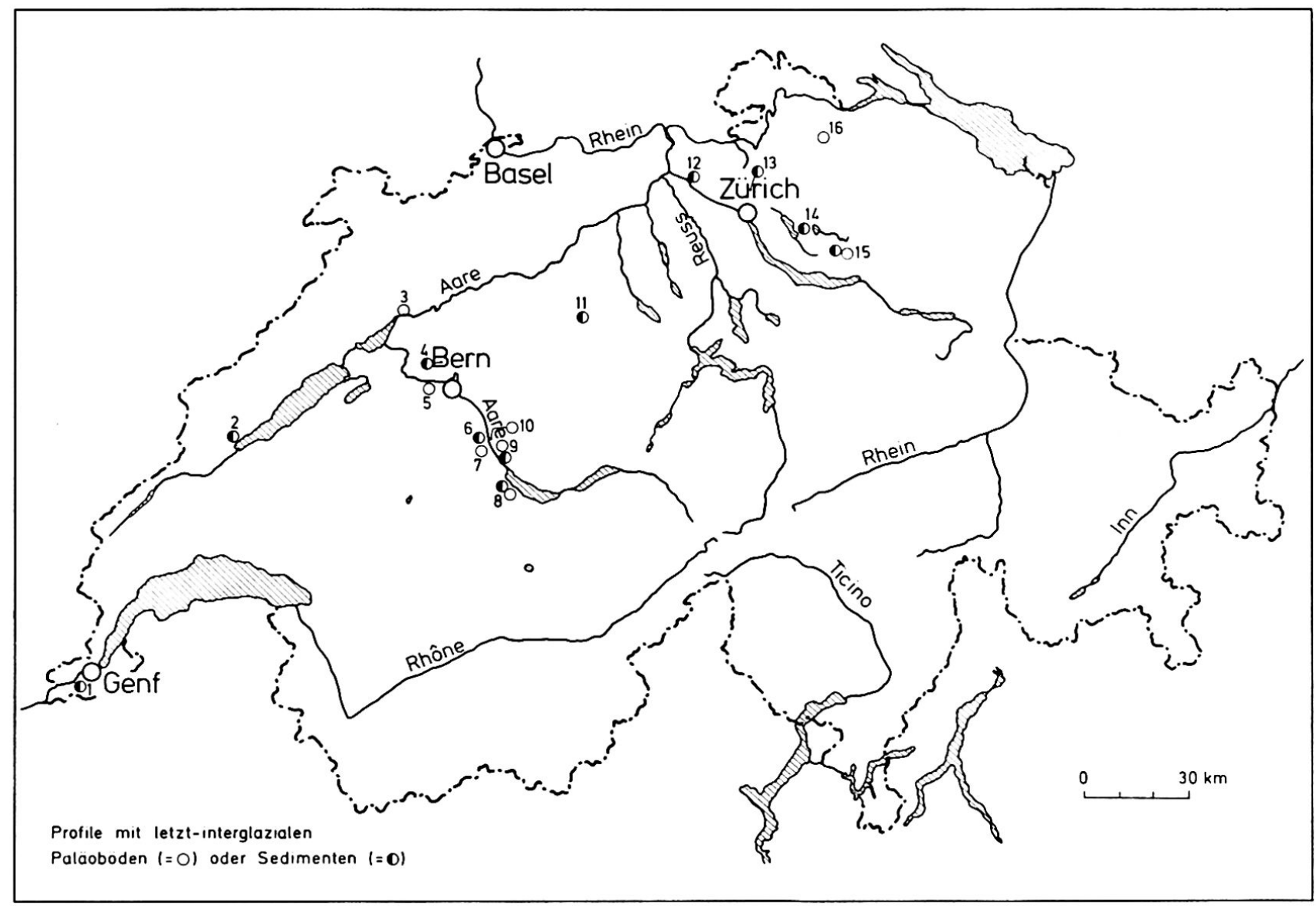

Abb. 5 Schematische Karte der Schweiz mit den diskutierten Interglazialvorkommen: $1=$ Confignon 1, 2 - Grandson, $3=$ Biel, 4 - Meikirch, 5 - Oberwangen, 6 - Thalgut, 7 - Jaberg, 8 - Glütschtal, $9=$ Bümberg/Thungschneit, $10=$ Wichtrach, $11=$ Gondiswil/Zell, 12 = Sulzberg, $13=$ Kloten, 14 = Uster, $15=$ Dürnten, 16 - Gütighausen/Thurtal.

eine stratigraphische Zuordnung der Thalgut-Seetone herangezogen werden, weisen ebenfalls auf älteres $(=$ interglaziales) Alter hin.

Die Bedeutung der Thungschneit-Seetone liegt neben der palynostratigraphischen Einstufung auch in der sedimentgenetisch kontinuierlichen Entwicklung aus den Liegendsedimenten, da dort die Seeablagerungen sich aus einem Eisrandtümpel direkt über Grundmoräne (der Raintal-Schlammoräne) entwickelt haben (SCHLÜCHTER, 1973).

Die stratigraphische Gliederung der Profile, welche palynostratigraphisch klassierbare Einheiten enthalten, ermöglicht im weiteren auch eine Absicherung der lithostratigraphischen Unterteilung. So ist es denn möglich, die in weiteren Profilen vorkommenden fossilen Bodenbildungen als wahrscheinlich zwischeneiszeitlich einzustufen: Jaberg, Wichtrach p. p., Bümberg p. p., Hurifluh und Alte Schlyffi p. p. (Abb. 5).

Wenn wir nun versuchen, über das engere Aaretal hinaus zwischeneiszeitliche Bildungen zu vergleichen, dann muß zuerst auf das Standardprofil Meikirch (WELTEN, 1981) hingewiesen werden, das in der Alpenvorlandsklimageschichte als Eem-Referenzprofil vorgestellt wird. Lithogenetisch sind die eemzeitlichen Pollenzonen an feinkörnige, sandig-tonige, geschichtete Silte gebunden (z.T. mit reichlichen pflanzlichen Makroresten).

Aus der Westschweiz sind bis jetzt mit Ausnahme der möglichen Profile von Grandson (weIdmanN, 1968) keine eindeutig letztinterglaziale Bezugshorizonte bekannt. Aus dem Genfer Becken beschreibt REYNAUD (im Druck) seine Einheit "Confignon 1" als möglicherweise interglazial, aber wahrscheinlich als Mindel/Riss der klassischen Chronologie. - Verwitterungshorizonte können in Plaffeien, Oberwangen bei Bern und in Biel (in Bohrungen der Seevorstadt) als interglazial interpretiert werden, doch fehlt vorläufig ein weiterer, absichernder biostratigraphischer Bezug. - Für die zwei palynostratigraphisch wichtigen Bezugsprofile von Gondiswil/Zell (durch PD Dr.S. WEGMÜLLER, Bern, in Bearbeitung) und vom Sulzberg bei Baden (welten, 1981), die detaillierte klimastratigraphische Information liefern, fehlt leider umgekehrt der weitere lithostratigraphische Bezug.

In der Ostschweiz gilt nun das Interglazialprofil von Uster (welten, 1981; wyssLing, 1978), zusammen mit den Resultaten aus der Gegend von Dürnten als Bezugsabfolge. Auch bei Uster ist die zwischeneiszeitliche Pollenzone an feingeschichtete, fossilhaltige Seetone gebunden. - Aus der weiteren Ostschweiz 




Abb. 6 Ausschnitt aus der Kiesgrube Jaberg mit den beiden letzteiszeitlichen Vorstoß-Schotter/Grundmoränenzyklen A/B und $C / D$. - A = Untere Aufschotterung, $B=$ Untere Grundmoräne und entsprechende Basisgroblage, $C=$ Obere Aufschotterung, $D=$ Obere Grundmoräne.

können vorläufig keine zusätzliche, sicher letztinterglaziale bereits beschriebene Sedimente erwähnt werden. Zwischeneiszeitliche Hinweise erhalten wir von einem kurzen Profil mit älteren Seetonen aus der Klotener Gegend (Baulos 8 oder Flughafenlinie) und durch einen fossilen Boden aus dem Thurtal östlich von Andelfingen (Kiesgrube Burghof bei Gütighausen). Teilweise ist das Interglazial auch in den Bohrungen von Dürnten durch einen Paläoboden belegt.

Aus Abbildung 5 geht die reliktische Verteilung der letzt-zwischeneiszeitlichen Ablagerungen und Bodenbildungen hervor. Gerade aus dem zentralen Mittelland erhalten wir aber genügend stratigraphische Information, um eine sedimentgenetische Entwicklung der letzteiszeitlichen Seidmente über dem interglazialen Bezugshorizont vorstellen zu können.

\section{Letzteiszeitliche Schlüsselprofile im Schweizer Mittelland}

Als Schlüsselprofile betrachten wir in diesem Zusammenhang solche, die einerseits einen klimastratigraphisch eindeutig faßbaren Horizont aufweisen; wo also zum Beispiel die letzte $Z$ wischeneiszeit belegbar ist und wo andererseits die Hangendsedimente mög- lic ist vollständig die Ereignisse der letzten Eiszeit dokumentieren. Solche Profile sind selten, und in der Regel schließen wir von komplizierten lithogenetischen Abfolgen auf deren "vollständige Ausbildung». Offen bleibt meistens die folgende Frage: wie sind die palynostratigraphisch gut definierten Profilabschnitte, vor allem auch die im Berner Querschnitt, lithostratigraphisch abgesichert?

\subsection{Thalgut}

Die letztinterglazialen Seetone (= Thalgut-Seetone) werden diskordant von sandig-siltigem $\mathrm{K}$ ies überlagert. Es handelt sich dabei um eine geringmächtige (bis max. $3 \mathrm{~m}$ ) «moränenähnliche Aufschotterung», in der auch die talseitige, glazitektonische Störung in den liegenden Seetonen ausklingt. Das Dach dieser Einheit ist verwittert (Cl-Horizont). Darüber folgt ebenfalls diskordant, und mit einer entsprechend ausgebildeten Basisgroblage, eine weitere, geringmächtige Aufschotterung, die dann ihrerseits durch die jüngste Grundmoräne eingedeckt wird. Im zentralen Kiesgrubenbereich sind jüngste Aufschotterung und Grundmoräne mächtiger entwickelt, wobei der liegende letzteiszeitliche Zyklus vollständig fehlt. 


\subsection{Jaberg}

Vergleichbare Verhältnisse wie im Thalgut finden wir in der Kiesgrube Jaberg. Der hier als letztinterglazialer Bezugshorizont ausgeschiedene, teilweise umgelagerte Paläoboden wird diskordant von einer bis mehr als $20 \mathrm{~m}$ mächtigen Aufschotterung überlagert, die ihrerseits von einer nur noch reliktisch erhaltenen Grundmoräne eingedeckt wird (Abb.6). Über diesem vollständigen Schotter-/Grundmoränenzyklus folgt mit einer einzigartig ausgebildeten Basisgroblage ein zweiter, ebenfalls vollständiger, Schotter-/Grundmoränenzyklus. Das doppelzyklische Jabergprofil über der letztinterglazialen Oberfläche ist sedimentgenetisch aus zwei Gründen wichtig: (a) die ältere letzteiszeitliche Aufschotterung überschüttet in mächtiger Art und Weise eine ältere Oberfläche und (b) die Basisgroblage der oberen Aufschotterung geht als Kondensationshorizont aus der Grundmoräne und den obersten Lagen der unteren Aufschotterung hervor (Abb.6). - Es handelt sich hier um eines der wenigen Profile, wo zwei letzteiszeitliche Schotter-/ Grundmoränenzyklen vollständig entwickelt sind: die Oberen Münsingenschotter sind hier in eine Obere und Untere Einheit weiter gliederbar.

\subsection{Bümberg/Thungschneit}

In der Kiesgrube Bümberg sind die letztinterglazial und früher tiefgründig verwitterten Schotter von Bümberg diskordant von den Münsingenschottern s. 1. überlagert, die teilweise mit einer ausgeprägten Basisgroblage, lageweise mit reliktischer Grundmoräne beginnen. Darüber folgt die jüngste Grundmoräne (= Rotachewald Grundmoräne). Im Thungschneitprofil folgen die letzteiszeitlichen Grobklastika ebenfalls diskordant über den interglazialen Thungschneit-Seetonen.

\subsection{Glütschtal}

Hier am Alpenrand sind die Profile im allgemeinen komplizierter aufgebaut als im nördlich gelegenen, «mittelländischen» Aaretal. Insbesondere sind viele Profile nur reliktisch ausgebildet (SCHLÜCHTER, 1973, 1976). Eine Erscheinung ist aber von grundsätzlicher Bedeutung: in den Profilen Hurifluh, Alte Schlyffi, Wässerifluh und Guntelsei sind zwischen den interglazialen Horizonten und den grobklastischen Aufschotterungen feinkörnige Stillwasserablagerungen mit den berühmten Schieferkohlen vorhanden. Es handelt sich dabei um eine lithostratigraphische Einheit, die nach dem palynostratigraphischen Befund eindeutig jünger als die letzte $Z$ wischeneiszeit ist und den mächtigen Aufschotterungen vorangeht. Diese Bildungen gehören hier zu den finalen Ablagerungen im Bereiche des letztinterglazialen Kander-/Simmedeltas in den Thunersee.

\subsection{Meikirch}

Die lithostratigraphische Gliederung der Sedimente über den interglazialen Seeablagerungen ist im einzelnen kompliziert: man ist gezwungen, die technische Qualität des Bohrkerns (1981) als genügend zu betrachten, auch wenn detaillierte Zuordnungen und Grenzziehungen nur grob möglich sind. Das Profil kann schematisch wie folgt beschrieben werden:

0- 9,65 m Grundmoräne mit rezentem Boden

$-12,00 \mathrm{~m}$ fluvioglaziale Schotter

$-14,30 \mathrm{~m}$ Grundmoränenkomplex

$-20,00 \mathrm{~m}$ feinkörnige Stausedimente mit Schotterlage von $15,00-18,00 \mathrm{~m}$

$-25,20 \mathrm{~m}$ sandige Schotter (verbohrtes Material)

$-33,00 \mathrm{~m}$ Grundmoräne (z. T. trocken)

$-38,45 \mathrm{~m}$ «Vorstoß-Schotter» mit Basisgroblage

$-39,30 \mathrm{~m}$ sandige (fluviatile ?) Schotter

im Liegenden: feinkörnige, geschichtete Seeablagerungen.

Ausgeprägte Hiaten oder Diskordanzen sind in der ganzen Abfolge nicht nachweisbar, und es ist nach den bisherigen Kenntnissen wahrscheinlich, daß die ganze hangende Abfolge über dem Eem genetisch als ein fluvioglazialer/glazialer Komplex aufzufassen ist. Zusätzliche sedimentologische Detailuntersuchungen sind noch im Gange.

\subsection{Uster/Glattal}

Die Sedimentabfolge in der Bohrung Nr. 221 bei Uster ist lithogenetisch für die Interpretation der letzteiszeitlichen Sedimente wichtig (nach L. \& G.WYSSLING, 1978): die interglaziale feinkörnige Serie wird von weiteren $59 \mathrm{~m}$ mächtigen, relativ monotonen siltigen bis sandigen Seeablagerungen überlagert. Nach wyssLING ist zwischen interglazialen und hangenden Seesedimenten kein Hiatus feststellbar, sondern der See, in dem die fossilführenden und jüngeren Sedimente abgelagert worden sind, hat ebenfalls über die ganze "frühe Zeit der letzten Eiszeit» bestanden. Unter geringmächtigen nacheiszeitlichen Sedimenten ist nur eine knapp $3 \mathrm{~m}$ mächtige Grund(?)moräne im Dach der Seesedimente vorhanden.

\subsection{Zürichsee und weitere übertiefte Talabschnitte}

Die im Sommer 1980 abgeteufte Bohrung ZüBO $80 \mathrm{im}$ Zürichseebecken hat Sedimente aufgeschlossen (LISTER, in Vorber.), die durchaus mit Ablagerungen in anderen übertieften Becken des Mittellandes verglichen werden können. Im Liegenden einer oft an sich schon schwer definierbaren jüngsten Grundmoräne sind mächtige, kaum gliederbare, feinkörnige, sandigtonige Silte mit einem unregelmäßigen Gehalt an Geröllen und Steinen, lageweise geschichtet, lageweise massig, vorhanden. - Aus Kernbohrungen im Seeland, dem Oberaargau und dem Aaretal sind solche Sedimente bekannt. Es scheint sich hier also um eine Fazies zu handeln, die den meisten übertief- 
ten Talabschnitten des Mittellandes gemeinsam ist. Die Präsenz des Gletschers während der Sedimentation, die Pollenarmut der Sedimente (oder zumindest ihr palynostratigraphisch schlecht definierter Inhalt) und ihr genetischer Zusammenhang mit grobkörnigeren, eigentlichen Grundmoränenlagen lassen an subglaziale Sedimentation in stehendes, strömungsfreies Wasser, zeitweise im Eiskontakt, denken (Schlammoräne oder waterlaid till [«Unterwassermoräne»], DREIMANIS, 1979). - Es handelt sich dabei um eine lithogenetische Einheit, die sich bisher als stratigraphisch schwer faßbar erwiesen hat, aber zur typischen Faziesentwicklung der letzteiszeitlichen (und älteren) Füllungen der übertieften Becken gehört. Wie am Beispiel der ZüBO 80 gezeigt werden kann (LISTER, in Vorber.), ist in dieser Situation der letztinterglaziale Bezugshorizont schwer nachweisbar beziehungsweise wahrscheinlich nicht (mehr) vorhanden.

\section{Lithogenetische Besonderheiten}

Aus den vorangehenden Kurzbeschreibungen geht hervor, daß am Aufbau der letzteiszeitlichen Akkumulationen vor allem zwei verschiedene Sedimenttypen beteiligt sind (abgesehen von den unzweifelhaft glazigenen Sedimenten s. str.): es sind dies (1) feinkörnige Stau- resp. Seeablagerungen, z.T. mit Schieferkohle, und (2) mächtige Schotterablagerungen. Dazu ist folgendes zu bemerken:

(1) Die feinkörnigen Stau- resp. Seeablagerungen sind aus zwei vollständig verschiedenen paläogeographischen Rahmen bekannt: einmal aus dem Glattal, wo nach der Bohrung 221 bei Uster (wYSSLING, 1978) über lange Zeiträume, vom Spätglazial der vorletzten Eiszeit bis zum Haupt(?)vorstoß der letzten Eiszeit, über die Zwischeneiszeit hinweg, ein See bestanden hat. Dort fehlen dementsprechend die anderswo mächtigen Aufschotterungen. - Dann sind ebenfalls aus dem Glütschtal feinkörnige frühletzteiszeitliche Sedimente bekannt. Dort stellt sich die Frage, ob diese Fazies an einen nach der Zwischeneiszeit höheren Thunerseespiegel gebunden ist oder ob es sich um Hochflutsedimente aus dem alten Kanderdelta handelt. Die ökologische Interpretation der Fossilien (Pelecypoda von der Wässerifluh) spricht für das Hochflutmilieu (sCHLÜCHTER, 1976).

(2) Die auffallendsten eiszeitlichen Ablagerungen außerhalb der heutigen Talrinnen sind die zum Teil mächtigen Aufschotterungen. Sie sind eigentlich das Hauptmerkmal der eiszeitlichen Alpenvorlandssedimentation. Ihre genetische Stellung in bezug auf eine Vergletscherung ist zum Teil aber immer noch kontrovers. - An Profilen, wie sie zum Beispiel im Aaretal oder im Seeland vorhanden sind, konnten großräumig die Beziehungen zwischen Aufschotterung und Gletschervorstoß untersucht werden. Dabei hat es sich gezeigt, daß die mächtigen talrandlichen Schotterkörper mit Grundmoräne in direkter genetischer Beziehung stehen und vorwiegend als VorstoßSchotter zu klassifizieren sind. Je nach präglazialer Topographie handelt es sich um gletscherrandliche bis frontale Sanderablagerungen, die dann vom gleichen Vorstoß überfahren worden sind (für Einzelheiten siehe SCHLÜCHTER, im Druck/*). In den meisten Talabschnitten des nördlichen Alpenvorlandes sind diese Aufschotterungen und deren fazielle Gliederbarkeit die einzige Grundlage, die für eine stratigraphische Rekonstruktion des eiszeitlichen Geschehens vorhanden ist (vgl. auch: KÄSER, 1980; KELLER, 1977, VAN DER MEER, 1982).

\section{Zusammenfassende Diskussion}

Der letztinterglaziale Bezugshorizont ist an wenigen Stellen durch Sedimente - Seeablagerungen oder Schieferkohlen - belegt (Glütschtal, Thungschneit, Thalgut, Meikirch, Uster, Dürnten, Sulzberg). Mit diesen palynostratigraphisch «datierten» Profilen können nun aber wiederum regional Paläoböden mit großer Zuverläßigkeit verbunden werden. Wohl sind diese Paläoböden in den meisten Fällen, vor allem wenn gut entwickelt wie zum Beispiel im Bümbergprofil, stratigraphisch mehrdeutig und können große Zeitspannen umfassen; doch sind sie als definierbare Hiaten wertvolle stratigraphische Bezugspunkte.

Die vorangehend schematisch dargestellten Profile erlauben die folgende stratigraphische Rekonstruktion der letzten Eiszeit: die Mittellandsprofile Meikirch und Uster lassen über dem Interglazial auf einen einzigen glazigenen Sedimentationszyklus schließen, wobei in Uster die Aufschotterung ganz fehlt und in Meikirch mächtig, aber zugleich faziell komplex ist. Ob auf Grund dieser Situation der Schluß gezogen werden darf, daß diese Gebiete seit dem palynostratigraphisch definierten Eem (sensu wELTEN) nur einmal vom Gletscher bedeckt worden sind oder ob es sich nur um unvollständige Profile handelt, ist noch nicht klar. Viele Argumente deuten auf vollständige Profile, also auf einmalige Gletscherbedeckung hin. Im gleichen Sinne können auch die Resultate von vaN DER MEER (1982) interpretiert werden (- im Berner Seeland sind die aufgeschlossenen Aufschotterungen nur bei Müntschemier in zwei Phasen gliederbar, wobei aber die liegende Einheit vorletzteiszeitlich sein kann). - Demgegenüber stehen die Resultate von KELLER (1977) und KÄSER (1980) aus dem Konfluenzbereich von Glatt, Töss, Thur und Rhein in der Nordostschweiz, wo doch wieder zwei «jüngere Aufschotterungszyklen» mit glazigenen Sedimenten vorhanden sind.

Die alpennäheren Profile im Aaretal Bern-Thun zeigen gegenüber den Mittellandsprofilen zwei vollständig ausgebildete fluvioglaziale/glaziale Akkumulationszyklen, und es stellt sich die Frage, ob sich aus 
diesen lithostratigraphischen Differenzen generelle paläogeographisch-regionale Entwicklungen ableiten lassen (vgl. dazu: SCHLÜCHTER, im Druck).

Die Frage der lithostratigraphischen Korrelation von talrandlichen Sedimenten und den Abfolgen in den übertieften Talabschnitten mußte bisher zum größten Teil offen bleiben. Die wissenschaftliche Kernbohrung (CS - SNF - 1), die im Auwald bei Kiesen im Rahmen eines Nationalfonds-Forschungsprojektes abgeteuft werden konnte (Juni 1981) hat für die Beantwortung dieser Frage eine wichtiges Resultat ergeben: zwischen 30,00 und $32,50 \mathrm{~m}$ ab OKT ist Grundmoräne (bis leicht verschwemmtes Grundmoränenmaterial) erbohrt worden, das als jüngste Grundmoräne innerhalb der heutigen Talfüllung vernünftigerweise mit der jüngsten, abdeckenden Grundmoräne der Umgebung (= Rotachewald Grundmoräne) korreliert werden kann. So können mit der Zeit auch die Füllungen der übertieften Talabschnitte stratigraphisch eingestuft werden; etwas, was heute immer noch mit vielen Fragezeichen geschehen muß.

\section{Literatur}

DIEGEL, F. (1975): Quartärgeologische Zusammenhänge im Jungpleistozän von Jaberg. In: Eclogae geol. Helv., 68/3: 517-530. Basel (Birkhäuser).

DREIMANIS, A. (1979): The problems of waterlain tills. In: SCHLÜCHTER, CH. (Ed.): Moraines \& Varves: 167-177. Rotterdam (Balkema).

HANTKE, R. (1978): Eiszeitalter. Die jüngste Erdgeschichte der Schweiz und ihrer Nachbargebiete, Bd. 1, Thun (Ott Verlag).

HANTKE, R. (1980): Eiszeitalter. Die jüngste Erdgeschichte der Schweiz und ihrer Nachbargebiete, Bd. 2, Thun (Ott Verlag).

KÄSER, U.J. (1980): Glazialmorphologische Untersuchungen zwischen Töss und Thur. - Dissertation, phil. Fakultät II, Universität Zürich. Zürich (Juris Druck \& Verlag).

KELLER, W. A. (1977): Die Rafzerfeldschotter und ihre Bedeutung für die Morphogenese des zürcherischen Hochrheingebietes. Vierteljahrsschr. Natf. Ges. Zürich, 122/3: 357-412. Zürich (Leemann).

LISTER, G. (in Vorber.): Results, discussion and interpretation from the sedimentological study of a long core through the basinal sediments and bedrock of Lake Zurich. Manuskript (in Vorber.).

MÜLLER-BECK, H.-J. (1959): Originalprofilaufnahmen Hurifluh und Wasserfluh. Unpubl. Ber. zuhanden Schweiz. Nationalfonds.

PENCK, A. \& E.BRÜCKNER (1909): Die Alpen im Eiszeitalter. Leipzig (Tauchnitz).

REYNAUD, Ch. (im Druck): Interprétation sédimentologique des dépôts Quaternaires du Pleistocène supérieur dans le sud du Bassin Genevois. Dissertation, Section des Sciences de la Terre, Université de Genève, Manuskript (im Druck).

SCHLÜCHTER, Ch. (1972): Geologie des Quartärs im Gebiet von Thungschneit bei Heimberg (Kt. Bern). Unpubl. Lizentiatsarbeit Univ. Bern.

\section{Dank}

Verbindlichst danke ich dem Schweiz. Nationalfonds zur Förderung der Wissenschaftlichen Forschung für das Ermöglichen einer Fortsetzung meiner Arbeit an den hier diskutierten Problemen im Rahmen des Projektes 2.698-0.80. Ganz besonders danke ich Herrn Prof. Dr. H. Oeschger und Frau T. Riesen, Universität Bern, für die Radiokarbondatierungen der Thalgutproben und für das Interesse an unseren Problemen. Verbindlichst danke ich ebenfalls Herrn Dr.H.-R. Keusen, Geotest AG Bern, für das Überlassen von neuem Bohrgut für eine wissenschaftliche Bearbeitung. - Sehr herzlich danke ich Herrn Prof.Dr. G. Furrer, Zürich, Herrn Prof.H.J. Lang, Zürich, Herrn Prof. Dr. E. Niggli, Bern, und Herrn Prof. Dr. M. Welten, Bern, für freundschaftliche und aufmunternde Kritik und für das meinen Untersuchungen entgegengebrachte Interesse. Ebenfalls herzlich danke ich Herrn PD Dr. F. Schweingruber, Birmensdorf, für die Bestimmung der fossilen Holzreste.

SCHLÜCHTER, Ch. (1973): Geologische Untersuchungen im Quartär des Aaretals südlich von Bern (Stratigraphie, Paläontologie, Sedimentologie). Unpubl. Diss. Univ. Bern (Juli 1973).

SCHLÜCHTER, Ch. (1975): Schotterpetrologie und deren relativ-stratigraphische Anwendbarkeit im Aaretal südlich von Bern (Schweiz). In: Eiszeitalter u. Gegenwart, 26: 74-81. Oehringen (Rau).

SCHLÜCHTER, Ch. (1976): Die lithostratigraphische Gliederung der letzteiszeitlichen Ablagerungen zwischen Bern und dem Thunersee. - In: FRENZEL, B. (Ed.): Führer zur Exkursionstagung des IGCP-Projektes 73/1/24 «Quaternary Glaciations in the Northern Hemisphere" vom 5.-13. Sept. 1976 in den Südvogesen, im nördlichen Alpenvorland und in Tirol. Stuttgart-Hohenheim (Manuskript).

SCHLÜCHTER, Ch. (im Druck/*): Genetic relationships between outwash deposits and basal till. Quaestiones Geographicae (im Druck).

SCHLÜCHTER, Ch. (im Druck): Das Maximum der letzten Eiszeit, dargestellt anhand der Verhältnisse im nördlichen Alpenvorland der Schweiz. - Kurzfassungen der Vorträge, DEUQUA-Hauptversammlung vom 1.-3.9.1982, Physische Geographie, Vol. 5, Zürich.

VAN DER MEER, J.J.M. (1982): Quaternary Geology and Holocene Soil Development in the area near Fribourg, Switzerland. Diss. Univ. Amsterdam. Publ. Fys. Geogr. Bodemk. Lab. Univ. Amsterdam, Nr. 32, 203 S.

WEIDMANN, D. (1968): Analyse pollinique dans les lignites quaternaires de Grandson. Travail de certificat inédit., Inst. botanique syst. et géobotanique, Univ. de Lausanne.

WELTEN, M. (1962): Arbeitsgemeinschaft zur Teilrevision des bernischen Quartärs. Unpubl. Ber. zuhanden Schweiz. Nationalfonds (7.Jan. 1962).

WELTEN, M. (1981): Gletscher und Vegetation im Lauf der letzten hunderttausend Jahre. Vorläufige Mitteilung. In: Jb. Schweiz. Natf. Ges., Wiss. Teil, 1978: 5-18. Basel (Birkhäuser).

WYSSLING, L. \& G. (1978): Interglaziale See-Ablagerungen in einer Bohrung bei Uster (Kanton Zürich). In: Eclogae geol. Helv., 71/2: 357-375. Basel (Birkhäuser). 